\title{
Alcohol Use and Gastrointestinal Disease
}

\author{
Helmut Karl Seitz ${ }^{\mathrm{a}}$ Sebastian Müller ${ }^{\mathrm{a}}$ Herbert Tilg $^{\mathrm{b}}$ Ali Canbay ${ }^{\mathrm{c}}$ \\ Eugen Zizer ${ }^{d}$

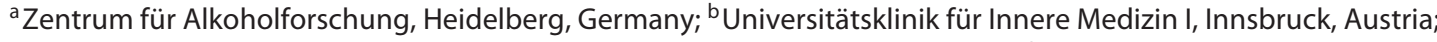 \\ ${ }^{c}$ Medizinische Universitätsklinik, Knappschaftskrankenhaus, Bochum, Germany; ${ }^{d}$ Klinik für Innere Medizin I, \\ Universitätsklinikum Ulm, Ulm, Germany
}

\section{Question 1: Has There Been Any Progress in Diagnosing Alcoholic Liver Disease during the Last 15 Years?}

Müller: Real progress in the early diagnosis of alcoholic liver disease (ALD) lies in the development of transient elastography. Pain-free and without any complications, early cirrhosis of the liver can be diagnosed within $5 \mathrm{~min}$ prior to severe complications.

Canbay: While the core diagnostics for patients with ALD have not changed much, some additional methods for detailed investigation have emerged.

The usual diagnosis starts with documentation of regular alcohol consumption, which has to be $>20 \mathrm{~g} /$ day for women and $>30 \mathrm{~g} /$ day for men in Germany to classify as ALD. These thresholds vary somewhat between countries and professional societies. This initial assessment should be followed by blood tests, to determine liver enzymes such as ALT, AST, and GGT. It is also necessary to exclude other potential liver diseases, such as hepatitis B or $\mathrm{C}$ or autoimmune hepatitis.

For a more detailed assessment of the disease state, transient elastography has become more established nowadays, to measure liver stiffness as a surrogate for liver fibrosis. Several attempts have been made to avoid liver biopsy by applying scores or noninvasive markers that could help in diagnosing the disease and/or its severity.
For example, the cytokeratin markers (cK18) M30 (caspase-cleaved cK18) and M65 (total cK18) have been tested. Even though both M30 and M65 are elevated in acute disease, they have also been shown to be elevated or to increase even further in abstinence. Except for transient elastography, no noninvasive diagnostic tools are available for ALD.

Regarding liver biopsy for diagnostic assessment, the trend has shifted toward mini-laparoscopy, which allows visualization of the liver surface prior to biopsy. In case of post-biopsy bleeding, it is possible to attempt to stop the bleeding under vision.

Finally, a good noninvasive marker has emerged to test for abstinence. Ethyl glucuronide is a long-term marker for alcohol consumption (for up to 6 months), which can be measured in hair, serum, or urine.

Tilg: The various stages of liver disease (especially early injury) can be better defined today, e.g., by the use of transient elastography.

Seitz: Yes, early alcoholic cirrhosis and even advanced fibrosis can be diagnosed with transient elastography at an early stage. In addition, various clinical scores predict prognosis in severe alcoholic hepatitis.

Zizer: In daily clinical life, laboratory scores have been established increasingly to judge the severity of ALD. His- 
tological evaluation is a diagnostic step used to diagnose ALD with certainty. However, the stage of fibrosis is one of the most important prognostic parameters in ALD and can now be easily determined using noninvasive methods such as Fibroscan or acoustic radiation force impulse imaging.

\section{Question 2: Is There Any Progress in the Treatment of Alcoholic Hepatitis?}

Müller: The treatment of severe alcoholic hepatitis is still a challenge. We know meanwhile that patients can be served through liver transplantation, but guidelines in Germany prohibit liver transplantation for these patients. Some few patients with a narrow window of indication benefit from steroids.

Canbay: The gold standard of treatment for alcoholic hepatitis has been corticosteroids. Several studies have been able to better define those patients who benefit from corticosteroid treatment. In the absence of infection and bleeding, corticosteroids will improve short-term survival, though mid-to-long-term survival will not be affected.

$\mathrm{N}$-acetylcysteine is an antioxidant which is reducing oxidative stress in hepatocytes. While given alone, $\mathrm{N}$-acetylcysteine does not affect the outcome, but when combined with prednisolone, it was able to improve survival and lowered the risk for infections and hepatorenal syndrome. Pentoxifylline, a phosphodiesterase inhibitor, did not improve survival, so it is no longer recommended.

Extracorporeal liver support can still only be considered a bridging therapy to transplantation and should only be performed in specialized centers. A big issue that is still difficult to tackle is treating/handling infections in patients with ALD.

Tilg: Supportive therapy has improved, but there is no real progress in the field of anti-inflammatory therapies.

Seitz: Not much progress has been made in the treatment of severe alcoholic hepatitis in the last decades. A major breakthrough was the fact that liver transplantation even without 6 months of abstinence showed similar long-term results to abstinence prior to transplantation. However, the public discussion is controversial due to the limited availability of organs and the fact that ALD is thought to be a self-imposed disease, which is only partially true.

Zizer: Studies of the last four decades have shown that the treatment of ALD has to be adjusted according to certain scores, such as the Maddrey Score or Lille Score. These scores help define those patients better who benefit from specific therapy, such as prednisolone and N-acetylcysteine. Malnutrition in patients with ALD has been focused on, and adequate enteral nutrition further determines prognosis for patients with severe alcohol-associated liver disease.

\section{Question 3: Is the Prognosis for Alcoholic and Nonalcoholic Fatty Liver Different?}

Müller: Exact comparable and long-standing prognostic data do not exist. When alcohol consumption is not stopped, the course of ALD is faster.

Canbay: Based on current clinical observations and evidence, the general prognosis for ALD, without any additional component of liver injury, is better than for nonalcoholic fatty liver disease (NAFLD). NAFLD is in most cases associated with metabolic comorbidities and a substantial risk of disease progression. The metabolic comorbidities - in particular, cardiovascular diseases and type 2 diabetes mellitus - are associated with higher mortality in NAFLD, with cardiovascular diseases as the main cause of death for NAFLD patients. ALD patients receiving liver transplantation have a fairly good long-term prognosis, given a sufficiently long duration of abstinence; in contrast, the prognosis for nonalcoholic steatohepatitisassociated liver transplantation is affected by the still present excess fat mass, leading to recurring NAFLD, and the abovementioned comorbidities. In reality, many patients present with overweight or obesity and resulting NAFLD with additional consumption of alcohol in varying amounts. It is virtually impossible to clearly separate NAFLD and ALD in cases with excess calorie uptake and alcohol consumption, with mutually aggravating liver injury and an enhanced disease risk.

Tilg: Progression of ALD is much faster and more dramatic (advanced liver disease, cirrhosis, alcoholic hepatitis, and hepatocellular carcinoma). The clinical feature of alcoholic hepatitis cannot be caused by NAFLD.

Seitz: ALD may progress faster than NAFLD. This process may depend on various factors including the amount of alcohol consumed, genetics, gender, and the presence of other liver diseases.

Zizer: The prognosis of certain factors is different in the two diseases. The amount of regularly consumed alcohol is one of the most important risk factors in alcoholic fatty liver. In NAFLD, deterioration of the fibrosis can be expected if arterial hypertension is present as a comorbidity. Common to both diseases is that patients who want to improve their health can greatly contribute 
to improving their prognosis. It has been shown that patients with a nonalcoholic fatty liver show significant improvement in the fatty liver with respect to inflammation, but also with respect to fibrosis, already after 1 year with an adequate diet and weight reduction of $10 \%$ of their total body weight. In patients with alcoholic fatty liver, abstinence is an important prognosis factor. Alcohol abstinence leads to a significant improvement of liver stiffness (Fibroscan) as well as to a significant improvement of portal hypertension (hepatic venous pressure gradient) after a short period of time already.

\section{Question 4: What Checkups Are Helpful for the Early Detection of Alcohol-Mediated Gastrointestinal Cancers?}

Müller: Sonography, elastography, gastroscopy, and colonoscopy.

Canbay: First of all, patients with alcoholic use disorder (AUD) and consequently a risk of ALD have to be identified: this is where primary care physicians play a very important role. Once the patients have been seen by a gastroenterologist/hepatologist, besides blood tests, ultrasound of the abdomen should be performed annually or biannually. Additionally, regular endoscopies (every 1-2 years if deemed necessary) of the upper and lower gastrointestinal tract should be performed. In case of suspicious areas, biopsies can be necessary. Screening for cancer markers in serum is not recommended.

Tilg: Liver ultrasound should be performed on all cirrhotics, and colonoscopy similarly as in the rest of the population.

Seitz: To detect cancer of the oropharynx and larynx, an ENT consultation, gastroscopy to detect esophageal cancer, and colonoscopy to detect polyps and colorectal cancer are recommended, as well as sonography of the liver.

Zizer: For gastroenterological alcohol-associated carcinoma (esophagus, colorectal, liver, and pancreas carcinoma), there are currently no specific screening programs. In addition, screening algorithms and current guidelines are not adapted to the profiles of patients with increased alcohol consumption. For example, patients with chronic pancreatitis receive regular sonographic or endosonographic checkups, irrespective of alcohol as a risk factor. In liver cirrhosis, regular sonographic checks are also recommended (every 6 months), but again, irrespective of the risk factor alcohol. However, there is increasing evidence that an adaptation of the surveillance/ screening program may be helpful for certain patients (genetic predisposition due to mutation of the PNPLA3 and/or TM6SF2 genes).

\section{Question 5: Do Genetic Factors Exist Which Favor the Development of Alcoholic Pancreatitis or Alcohol-Mediated Pancreatic Cancer?}

Müller: Some genetic factors are associated with alcoholic pancreatitis, but the association is weak, complex, and therefore not helpful for screening.

Canbay: There are only few studies on this topic and still many open questions in this field. Overall, it is not understood at all why one patient with an AUD will develop pancreatitis and another patient with AUD will develop ALD. It is estimated that $50 \%$ of AUD development is due to genetics. AUD is the most common cause of development of chronic pancreatitis. Chronic pancreatitis can lead to the development of pancreatic cancer in the worst-case scenario.

There are genetic factors that can be linked with pancreatitis (e.g., the CFTR gene or the SPINK1 gene) or pancreatic cancer $(B R C A)$. A general screening of families should, as always, be recommended with caution.

Tilg: Yes, at least for the development of alcohol-associated pancreatitis, there are several genetically backed associations, such as CLDN2-MORC4, CTRC, PRSS1PRSS2, and SPINK1; last rediscovered has been CTRB1CTRB2 (chymotrypsin B1 and B2).

Seitz: Yes, they do, but the data are limited, and the association seems not to be strong.

Zizer: In the past, multiple genetic factors associated with alcoholic chronic pancreatitis have been described. These are risk genes as well as protective genes; thus, the PRSS1-PRSS2 rs10273639 T allele plays a protective role with respect to the development of alcoholic chronic pancreatitis. In this context again, RIPPLY1 (rs7057398) as well as CLDN2-MORC4 (rs12688220) polymorphisms are predisposing genes. Furthermore, SPINK1 mutations can be found frequently in patients with alcohol-induced pancreatitis.

\section{Question 6: What Are the Causes of Alcohol-Associated Diarrhea?}

Müller: This has not been studied extensively. Alcohol may directly injure the mucosa and may change muscle contractions and the microbiome. 
Canbay: There are multiple reasons for alcohol-induced diarrhea. Besides AUD and alcoholic hepatitis, when drinking too much alcohol, the mucosa of the small intestine is changed temporarily. Through alcohol consumption, sodium channels are blocked, so neither sodium nor, consecutively, water can cross the mucosal barrier, resulting in more liquid retained in the intestine, hence leading to diarrhea.

In patients with AUD, the gut microbiome can be a factor for development of diarrhea. If most calories are taken in by alcohol consumption and not food, the microbacterial environment of the intestine changes. Thus, the absorption of nutrients such as fat can be permanently disrupted, causing diarrhea.

Tilg: Partly there are probably direct effects on different ion channels in the epithelial cells.

Seitz: Alcohol-mediated mucosal injury, motility disturbances, enhanced bile flow, colonic water secretion due to alcohol-mediated stimulation of mucosal cyclic AMP, and a change in the colonic microbiota.

Zizer: In this context, various factors play a role. On the one hand, alcohol consumption changes the intestinal microbiome, shifting the balance towards proinflammatory bacterial species (more proteobacteria, fewer Bacteroidetes). Another factor is the alcohol-induced disturbance of the mucosal barrier function. This is especially true in the small intestine, with consecutive disturbance in the absorption of water and sodium. In addition, patients with alcohol-associated diarrhea have an increased transit in the small intestine, possibly due to toxic damage to the mucosa of the small intestine as well as alterations in intestinal neuronal regulation.

\section{Participants}

Prof. Helmut Karl Seitz

Zentrum für Alkoholforschung

Im Neuenheimer Feld 365

DE-69120 Heidelberg (Germany)

helmut_karl.seitz@urz.uni-heidelberg.de

Prof. Sebastian Müller

Zentrum für Alkoholforschung

Im Neuenheimer Feld 365

DE-69120 Heidelberg (Germany)

sebastian.mueller@urz.uni-heidelberg.de

Prof. Herbert Tilg

Universitätsklinik für Innere Medizin I

Anichstrasse 35

AT-6020 Innsbruck (Austria)

Herbert.Tilg@i-med.ac.at

Prof. Ali Canbay

Medizinische Universitätsklinik

Knappschaftskrankenhaus

In der Schornau 23-25

DE-44892 Bochum (Germany)

Ali.Canbay@ruhr-uni-bochum.de

\section{Dr. Eugen Zizer}

Leiter Schwerpunkt Hepatologie

Klinik für Innere Medizin I

Universitätsklinikum Ulm

Albert-Einstein-Allee 23

DE-89081 Ulm (Germany)

eugen.zizer@uniklinik-ulm.de

\section{Disclosure Statement}

H.K. Seitz, S. Müller, H. Tilg, and E. Zizer have nothing to disclose. Ali Canbay received payments for consultancies, expert testimony, or lectures from Merz Pharma Gmbh \& Co. KGaA, Takeda Pharma, Sanofi, Dr. Falk Pharma GmbH, and Gilead. 